FERMILAB-Conf-88/33

\title{
Experimental Possibilities for Observation of CP Violation in B Decay*
}

\author{
Brad Cox \\ Fermi National Accelerator Laboratory \\ P.O. Box 500, Batavia, Illinois 60510
}

February 1988

*Invited talk delivered at the International Symposium on Heavy Flavors, Stanford University, September, 1987 


\title{
Experimental Possibilities for Observation of CP Violation in B Decay*
}

B. Cox

\begin{abstract}
The detection and the measurement of $\mathrm{CP}$ violation in the decays of $\mathrm{B}$ hadrons will require the accumulation of large numbers of $B$ decays in particular exclusive modes. The potential of beauty hadroproduction experiments for obtaining these large data samples is examined in the context of various trigger and analysis strategies. The possibilities for doing such experiments at present hadron machines is compared with the potential for such experiments at the SSC.
\end{abstract}

*Invited talk delivered at the "International Symposium on Heavy Flavors", Stanford University,September, 1987 


\section{Introduction}

The accumulation of large numbers of B decays for purposes of observation and measurement of $\mathrm{CP}$ violation effects has recently become a topic of great interest in the high energy physics community. Indeed, B decay may be the only place other than the $\mathrm{K}^{0}$ system where $\mathrm{CP}$ can be observed experimentally and, as such, may present the our only other experimental possibility for understanding the origin of $\mathrm{CP}$ violation. The number of produced B's necessary to observe $\mathrm{CP}$ violation depends on the strategy employed and the decay mode in which the observation is attempted, but it is thought that an experiment must produce at least $10^{7} \mathrm{~B} \mathrm{~B}$ 's to have the possibility of detecting $\mathrm{CP}$ violation. This is partly because the cross sections for $\mathrm{B}$ production and partly because the branching ratios for particular exclusive modes are small. In addition, the separation of the $B$ decays into particle and antiparticle data samples, a prerequisite for searching for $C P$ violating effects, will require that the statistics of the $B$ decay data be larger than is otherwise be necessary for studying other aspects of B physics. Hence, while the asymmetries between $\mathrm{B}$ and $\mathrm{B}$ decays into particular exclusive final states are expected to be large,the detection of $\mathrm{CP}$ violation presents a formidable experimental problem.

While hadronic experiments have historically been less successful in performing spectroscopy than $\mathrm{e}^{+} \mathrm{e}^{-}$experiments because of the large hadronic total cross sections that must be handled, the cross section for beauty production is considerably larger in hadronic interactions than in $\mathrm{e}^{+} \mathrm{e}^{-}$interactions'. Because of the larger cross sections, obtaining large enough numbers of B's for $\mathrm{CP}$ measurements seems much more feasible in hadroproduction experiments than in $\mathrm{e}^{+} \mathrm{e}^{-}$ experiments. Therefore, the necessity of producing large numbers of B's for CP violation experiments has generated great interest in finding experimental techniques and strategies for hadronic experiments that would allow the separation of BB events from the total cross section.

A combination of the development of the technologies described in Section III and the unique features of the $B$ hadrons give hope that experiments can be performed which can obtain the large data samples required. In particular, the long lifetime $2\left(1.42 \pm 0.27 \times 10^{-12} \mathrm{sec}\right)$ of the $B$ hadrons, the recently observed large mixing ${ }^{3}$ of the $\mathrm{B}^{0}{ }_{\mathrm{d}}$ meson and the report of a charmless $\mathrm{B}$ decay $\left(\mathrm{B}_{\mathrm{d}}>\overline{\mathrm{p}} \mathrm{p} \pi \pi\right)$ by the ARGUS collaboration greatly enhance the chances of observing $\mathrm{CP}$ violation effects in $B$ decays. The purpose of this paper is to describe experimental techniques and strategies that can be employed in hadronic experiments to obtain large, relatively clean B decay 
samples and to outline the steps that must be followed to observe $\mathrm{CP}$ violation in the data.

\section{Ih. Beauty Hadroproduction Cross Sections}

While the possibility for obtaining large numbers of B decays in hadronic experiments is intriguing, the present experimental situation in the observation of $\mathrm{B}$ decays in hadronic interactions makes it obvious that we are far from realizing this potential. To date only two $\mathrm{B}$ decays have been reconstructed in a hadroproduction experiment ${ }^{4}$, WA75. The situation is scarcely better in $\mathrm{e}^{+} \mathrm{e}^{-}$ experiments where the reconstructed $B$ decays number less than a few hundred events ${ }^{5}$. In addition, there are to date only a few experiments which have reported ${ }^{0,7,8.8}$ indirect measurements of the hadroproduction cross section for B's. Estimates of the cross section of B production in $320 \mathrm{GeV} / \mathrm{c} \pi^{-} \mathrm{U}$ interactions have been made by WA78 from measurements of the production of trimuons ${ }^{6}$ and same sign dimuons ${ }^{7}$. From these measurements, the WA78 experimenters have extracted a $\pi^{-}$nucleon cross section (assuming a linear A dependence) of $(2.0 \pm 0.3 \pm 0.0) \mathrm{nb}$ per nucleon. The UA1 collaboration ${ }^{8}$ has inferred from the high $\mathrm{p}_{\mathrm{t}}$ dimuon events in their $\sqrt{\mathrm{s}}=639 \mathrm{GeV} \mathrm{p} \overline{\mathrm{p}}$ data a $\mathrm{B}$ hadroproduction cross section of $1.2 \pm 0.1 \pm 0.2 \mu \mathrm{b}$ for the portion of the cross section at $p_{t}>5 \mathrm{GeV} / \mathrm{c}$.

To estimate the pp cross section for B production at TEV II (Fermilab fixed target mode) and the $p \bar{p}$ cross section at TEV I (Fermilab collider mode), the calculations of Ref. 10 have been used. The parameters (for example, the b quark mass, large variable,etc.) for these calculations have been chosen to achieve agreement with the WA78 and UA1 results. This procedure yields a pp hadroproduction cross section of $8 \mathrm{nb}$ for TEV II experiments at $\sqrt{\mathrm{s}}=41 \mathrm{GeV}$ and a p $\bar{p}$ cross section for TEV I of approximately $15 \mu \mathrm{b}$ at $\sqrt{\mathrm{s}}=2000 \mathrm{GeV}$. At SSC energies $(\sqrt{\mathrm{s}}=40000$ $\mathrm{GeV}$ ) the same type of calculation" 1 has been used to estimate a pp cross section. Because of the

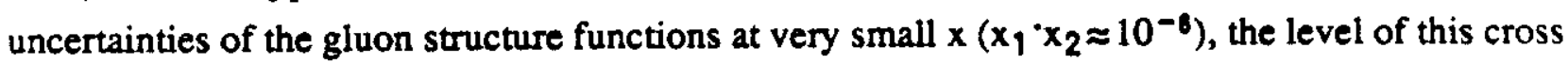
section is quite uncertain. At present time, the estimate can range anywhere between $100 \mu b$ and 1 mb. We take the lower value as the conservative choice for purposes of comparisons between machines. The pp total cross sections are taken to be $32 \mathrm{mb}$ at $\sqrt{\mathrm{s}}=41 \mathrm{GeV}$ and $100 \mathrm{mb}$ at $\sqrt{\mathrm{s}}$ $=40000 \mathrm{GeV}$ and the $\mathrm{p} \overline{\mathrm{p}}$ cross section at $\sqrt{\mathrm{s}}=2000 \mathrm{GeV}$ is taken to be $100 \mathrm{mb}$ for purpose of estimating trigger rates. For purposes of calculating B B yields at TEV $\Pi$, we have assumed the use of intermediate $A$ targets for fixed target experiments, leading to enhancements of 2 to 3 of the $\sqrt{\mathrm{s}}=41 \mathrm{GeV} \mathrm{pp} \rightarrow \overline{\mathrm{BB}}$ hadroproduction cross section (assuming a linear A dependence for the $\mathrm{B}$ 
cross section).

Using these beauty cross section estimates, the numbers of B mesons produced in $10^{7}$ seconds of operation of each machine are shown in Table I along with the ratio of $B$ hadroproduction cross section to total cross section for each machine. The assumption implicit in this table is that both TEV II and the SSC are limited not by the available beam flux but by detector or data acquisition system to operate at $10^{7}$ interactions per second. The TEV I limitation is taken to be available machine luminosity (approximately $10^{31} \mathrm{~cm}^{-2} \mathrm{sec}^{-1}$ if a TEV I upgrade is implemented). The duration of experiments for each configuration is taken to be $10^{7}$ seconds at peak intensity.

In addition to estimating the numbers of events that may be obtained in a $10^{7}$ sec run in various experiment configurations, the features of the production distributions of these events must be taken into account. These distributions will significantly affect the design of any spectrometer aspiring to measure $B$ hadron decays. In addition, the triggering on the $B$ events and the reconstruction of the $B$ hadrons with good resolution off line will be significantly affected by these distributions. It has been pointed out ${ }^{12.13 .14}$ that $b$ quark production, which is dominated at SSC energies by gluon fusion, has a striking signature which makes the SSC B event topologies look very much like the Lorentz boosted $B$ hadrons produced in Fermilab fixed target experiments. Therefore, a beauty spectrometer configuration at the SSC and in fixed target experiments can be both forward and have relatively small acceptance.

Using the PYTHIA hadroproduction Monte Carlo ${ }^{15}$ based on the Lund string model, we have studied the distributions of the $b$ and $\bar{\sigma}$ quarks (as well as the $B$ hadrons resulting from the $b$ quark hadronization). The first significant observation is that there is a strong peaking along either beam direction for the $b$ or 5 production in both the SSC and the TEV I colliders. This peaking is significantly less pronounced in TEV I interactions. The TEV II B events are, of course, strongly peaked forward because of the Lorentz boost. In addition, there is a strong correlation with momentum such that only the b's emitted along beam directions have large momentum. The correlation of momentum with production angle is shown in Fig la for the SSC, TEV I and TEV II. Notice that the b quarks produced at TEV II and the SSC have considerably higher momentum on average than do the $b$ quarks produced at TEV $I$. In addition, the centrally produced $b$ quarks are quite low in momentum at the colliders. The hadronization of the $b$ quarks at the higher 
energies of the colliders further decreases the average momentum of the resulting B hadrons so that TEV II in the end has higher energy B hadrons than even the SSC. This results in the decay products of the B hadrons at TEV II having higher momentum than the collider B's even in the forward region along the beam. As an example, the average momentum of the electons from semileptonic B decays is shown in Fig. 16 for TEV II, TEV I and the SSC.

There is also a striking correlation of the $b$ and $\bar{\sigma}$ quark production angles at the colliders such that both the $b$ and $\bar{b}$ quarks are produced in the same direction aligned with one of the beams. The Lorentz boost in fixed target experiments will produce qualitatively the same effect. These correlation is shown in Fig. 2a,b and $\mathrm{c}$ for the SSC and TEV I and TEV II. It is apparent that gluon fusion at the higher energies of the colliders produces distributions which are qualitatively similar to the effects of a Lorentz boost in a fixed target experiment at $\sqrt{\mathrm{s}}=41 \mathrm{GeV}$. Because of this, a spectrometer designed specifically for detecting beauty at the SSC would look quite similar to a fixed target experiment ${ }^{11,12,13.15}$.

We have given in Table I below a comparison of the event rates and some average quantities for the B events at TEV II, TEV I and the SSC. By the naive criterion of total produced events per experiment, all three experimental configurations can produce events at a rate greater than the $10^{\prime}$ events per experiment deemed necessary for $\mathrm{CP}$ violation measurements. However, specific strategies must be evaluated one by one to estimate the level of sensitivity that each configuration has for detecting $\mathrm{CP}$ violation effects.

\section{Experimental Detection of B's}

The development of three technologies in the last decade has made the $B$ hadroproduction physics experimentally accessible. Since the ratio of B hadroproduction cross section to total cross

section varies from $\approx 10^{-6}$ in TEV II to $10^{-3}$ at the SSC, powerful techniques for separation of the B decays from the rest of the total cross section both at trigger time and in the off line analysis are essential. The three technological developments that allow this to be accomplished experimentally are:

1. The development of silicon microstrip trackers which can resolve secondary vertices separated by less than a few 10 's of microns from the primary production 
vertex.

2. The development of fast triggers and fast trigger processors which, on the time scale of microseconds, can do relatively complicated calculations and make sophisticated decisions to separate B events from the total hadroproduction cross section events.

3. High rate data acquisition systems which can deliver a megabyte of data to tape per second.

The combination of these three developments and the unique physics attributes of the $B$ hadron decays make it possible to consider separating B production from the much larger total cross section. The relatively long $B$ decay lifetime $\left(\approx 1.42 \times 10^{-12}\right.$ seconds) results in decay paths which, as indicated in Table I, average 7000 microns at TEV II, 2000 microns at TEV I and 3000 microns at the SSC. The longer decay paths at TEV II highlight the interesting fact that the highest momentum B's are produced at TEV II, not at TEV I or even at the SSC. Primary and secondary vertices separated by these sorts of distances are can be resolved with good efficiencies using the silicon tracker technology. By contrast in $e^{+} e^{-}$interactions at the $4 S$ resonance, the $B$ and $B$ are produced essentially at rest with respect to the overall interaction, and their decay products must be untangled without aid of a distinct secondary vertex. Thus, resolvable secondary vertices in hadronic interactions allow the experimenter to avoid the combinatorial problems that would be encountered if a search through all possible track combinations had to be attempted.

\section{Trigger Strateries}

The process of separating at the trigger level the beauty signal from the huge number of interactions due to the hadronic total cross section requires the development of sophisticated trigger strategies that can be implemented in multilevel trigger hardware. The proposed interaction rate of $10^{7}$ interactions per second at TEV II and the SSC must be reduced by the trigger to $<10^{2}$ events per second to match the capabilities of data acquisition systems. TEV I has lower rates but a more difficult trigger problem because of the relatively low momentum of the B's in Fermilab collider compared to either the SSC or the Fermilab fixed target configurations. In all experimental configuration (as indicated by Table I), the rate of events containing a B B is a small percentage of 
the total interaction rate.

In considering the general problem of how to eliminate the interactions due to the total cross section while preserving the B B events, an experiment can choose to implement a "physics" trigger on either the particular B decay that is used to tag the particle/antiparticle identity of the second $\mathrm{B}$ in the event or on the specific $\mathrm{B}$ exclusive decays that are to be be analysed for evidences of CP violation. Hereafter, we will designate the B's in a given event as either the "tag" or the "analysis" B depending on its role in the experiment. Presuming that one needs to tag, then the triggering procedure is symmetric except for the relative rates of the "tag" B's and the "analysis" B's. For some types of tagging (for example, a tag which depends on the charge of the lepton from the B semileptonic decays), the rate of "tag" B decays may be quite large compared to the rate of particular "analysis" B exclusive modes. If the large rate can be handled, it may be better to trigger on the tag B (ignoring asymmetries that may be, therefore, built in at trigger time) and try to simultaneously accumulate data on several exclusive modes. With the "tag" B decay in hand the resulting sample of events can be sifted for specific exclusive modes of the "analysis" B in which $C P$ violating effects may be observed.

On the other hand, the trigger rates of tagging decays may be so large that they may limit an experiment's ability to accumulate enough data in the particular exclusive channels where the CP effects are large. An example of this problem is the the fairly loose trigger based the semileptonic modes of the B where the charge of the resulting lepton is used to tag the other B in the event as B or B (see Section VI). In an SSC experiment, the data acquisition system may be clogged at $10^{7}$ interactions per second by the tag B semileptonic triggers alone even without including the triggers due to $\pi$ and $\mathrm{K}$ semileptonic decays. In such cases, a more restrictive,"double smart" trigger aimed specifically at a relatively rare "analysis" B decay may be a more desirable strategy. Such a trigger could not only select particular B exclusive modes which are expected to exhibit large CP violation but also have better rejection of backgrounds. Because of this, triggering on the "analysis" B may be a more optimal way to proceed if the trigger rate and data acquisition system limits the experiment. The events thus obtained could then be searched for the other B decay in the hope of obtaining the particle or antiparticle tag by means of one of the several techniques discussed in Section VI

Finally, an experiment can, of course, trigger on both the "tag" and the "analysis" B 
simultaneously if the rate of both "analysis" and "tagging" decays are both too large independently. Only careful estimates can determine what is the proper strategy for a particular experiment.

In addition to the physics triggers discussed above, a number of triggers have been proposed which are "generic". These triggers do not select particular decay modes of either the tagging or analysis B and, therefore, do not prejudice the physics before hand. An example of such a trigger is one which requires at trigger time the presence of a secondary vertex in the interaction. This type of trigger does not specify either the tag or analysis B decay except in a second order way through the details of how the secondary vertex trigger is implemented.

At present, a list can be made of several triggers which have been discussed as possibilities for selecting B events. The effectiveness and feasibility of any of these trigger schemes is still in question. The proper trigger procedure will vary from experiment to experiment and will depend on the ultimate objective of the experiment. Indeed, the proper procedure will probably involve a mixture of several different triggers in most cases to achieve the $10^{-3}$ to $10^{-6}$ rejection of total cross section that is needed to match the capabilities of the data acquisition systems. The triggers that have been most discussed are:

1. Triggering on the dilepton from the $B->J / \Psi+x$ decays $12.13 .17 \quad$ "Physics" Triggers

2. Triggering on the single lepton "tagging" semileptonic $B$ decay ${ }^{18.19}$

3. Triggering on the presence of a secondary vertex ${ }^{20.21}$

4. Triggering on the presence of several moderate $p_{t}$ hadrons ${ }^{22}$

"Generic" Triggers

Space does not permit the discussion in detail of all of these trigger strategies with their attendant tagging strategies. As an example, a trigger strategy based on the B->J/ $\psi$ inclusive decay mode will be outlined below to demonstrate various facets of the triggering and analysis problems.

\section{VThe $I / \Psi$ Strategy}

The J/ $\Psi$ trigger strategy is based on the measurements by the ARGUS $(1.17 \pm 0.16 \pm 0.22 \%)$ and CLEO $(1.09 \pm 0.16 \pm 0.21 \%)$ collaborations of an appreciable rate ${ }^{23.24}$ for the inclusive decay, $\mathrm{B}^{0}{ }_{\mathrm{u}} \rightarrow \Psi+\mathrm{x}$. The best estimate, combining both experiments, is taken to be $1.13 \%$ for this mode. 
If we specialize the discussion of this strategy to the dimuon decay of the $\mathrm{J} / \Psi$ (although it is also possible to consider dielectron decays), the combination of the B->J/ $\Psi$ branching ratio with a branching ratio for $J / \psi->\mu^{+} \mu^{*}$ of $0.07 \pm .01$ yields a rate per second for the process

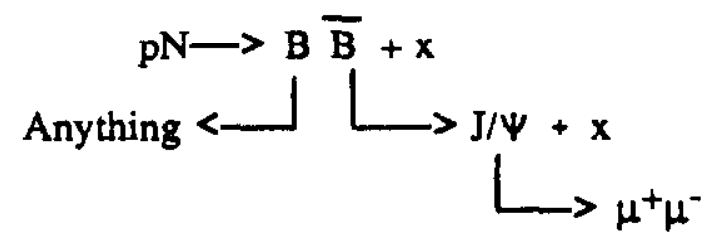

proportional to (allowing for two B's per event) $2 \times 7.9 \times 10^{-3} \times$ ratio of B production cross section to total cross section $\times$ operating interaction rate for TEV II,TEV I or the SSC.

Even though the composite branching ratio for the $B->J / \Psi \cdot>\mu^{+} \mu^{-}$process is small compared to the branching ratio for the decay, B-> $\mu v+$ hadrons (the semileptonic decay has a rate of $1.1 \times 10^{-1}$, approximately 130 times the $\mathrm{B}->\mathrm{J} / \Psi->\mu \mu$ composite branching ratio), the $\mathrm{J} / \Psi$ mode is a particularly nice approach to the problem of isolating a definitive beauty signal from hadronic backgrounds for several reasons:

- The observation of a $\mathrm{J} / \Psi$ at a secondary vertex is an unambiguous signature of a beauty decay. Unlike the semileptonic mode, it cannot be faked by a charm decay.

- The backgrounds to this decay sequence are relatively tractable. The major background are $\mathrm{J} / \Psi$ 's produced in the primary interaction but mismeasured because of multiple scattering in the silicon tracker. In order to contribute to the background to the $J / \Psi$ 's from B decay, both muons from the mismeasured primary vertex $\mathrm{J} / \Psi$ must appear to be associated with one another (i.e., have a small distance of closest approach) and form a secondary vertex at an appreciable distance from the primary vertex. In addition, this scattered $\mu$ pair would have to form a $J / \psi$ mass. Without requiring the reconstructed $\mu$ pair mass to be that of a $J / \Psi$, the Fermilab E771 experimenters ${ }^{17}$ estimate that the probability for two muons from directly produced $\mathrm{J} / \Psi$ to have their point of closest approach at greater than 50 microns from the reconstructed vertex in the plane transverse to the beam 
direction and greater than $1 \mathrm{~mm}$ along the beam in a $900 \mathrm{GeV} / \mathrm{c} \mathrm{pN}$ interactions to be less than $3 \times 10^{-5}$. The additional requirement that a third particle intersect (within 50 microns in the transverse plane) with the faise $\mathrm{J} / \Psi$ secondary vertex will increase the rejection of mismeasured $\mathrm{J} / \Psi$ 's by a factor $>10$. If the same resolutions were obtainable in a collider configuration, the rejection of directly produced $\mathrm{J} / \Psi$ 's becomes easier since the ratio of $\mathrm{J} / \Psi$ resulting from $B$ decays to directly produced $\mathrm{J} / \Psi$ increases from $10^{-3}$ at TEV II to few $\times 10^{-1}$ at SSC energies. However, since the B's at SSC and TEV I (according to Table I) will have considerably lower momenta, it may not be feasible to achieve the same vertex resolution at the colliders, making background rejection harder from that standpoint.

- The muon pair is a relatively easy signature to trigger on. Backgrounds to the dimuon trigger are due to $\mathrm{K}$ and $\pi$ semileptonic decays into muons and to punch through of hadrons in the muon detector. The semileptonic decays appear to dominate the trigger. Estimates done for TEV $\Pi^{17}$ and for the $\mathrm{SSC}^{13}$ indicate that the trigger rates can be achieved that are roughly compatible with the present capabilities of data acquisition systems (approximately 1 megabyte per second of data in to permanent storage ${ }^{25}$ ). It is worth pointing out that there will be approximately $10^{3} \mathrm{~b} \sigma$ 's produced per second at a luminosity of $10^{32} \mathrm{~cm}^{-2} \mathrm{sec}^{-1}$. If the $b \bar{b}$ event size is taken to be a relatively modest 5000 bytes (present TEV II fixed target event sizes are somewhat larger than this), a perfect trigger that selected all of these and only these events would require a data acquisition system that could handle greater than 5 megabytes of data per second. Of course, unavoidable trigger backgrounds will increase any such perfect trigger rate by a large factor. So the choice of a more restrictive trigger on a rarer process such as $B->J / \Psi->\mu \mu$ for an experiment at the SSC may yield as many events as one based on the more abundant $B$ decay modes, due to the combination of higher trigger rates for the less restrictive triggers and limitations of data acquisition systems.

o The $B->J / \Psi+x$ inclusive decay mode is expected to encompass a 
relatively high percentage of exclusive decay channels which 1) have only one or two other particles in addition to the $J / \Psi$ and 2) can be totally reconstructed because the other particles are all charged. The relatively small number of additional particles in the $J / \Psi$ decays is due in part to the limited phase space and the fact that the charmed quarks are locked into the $\mathrm{J} / \Psi$ and, by definition, required to decay into a $\mu$ pair. This serendipity of exclusive modes is demonstrated in Table II below:

- The focus on the $\mu$ pair gives a clear cut way of proceeding in the search for secondary vertices in the trigger sample. This procedure, since it starts with the two muons, is relatively simple since all track combinations in the event do not have to be examined for evidence of a secondary vertex.

- Finally, the $B->\Psi+x$ decay modes contain several good prospects for observation of $C P$ violation among which are the $B->\psi \mathrm{K}^{0}{ }_{\mathrm{s}}, \mathrm{B}->\psi \pi^{+} \pi^{-}$, and $\mathrm{B}->\psi \varphi$ modes. These particular decays result in final states which are $\mathrm{CP}$ eigenstates and, as such, have the particularly simple and elegant $\mathrm{CP}$ violation signatures in the time distributions as discussed in Section VIII.

Table II lists only modes without $\pi^{0} \mathrm{~s} . \mathrm{K}^{\mathrm{O}} \mathrm{s}$ are considered less difficult to handle in the reconstruction of the B's since the average multiplicity of this species is low enough to present no combinatorial problem.

\section{VL. Tagging the Particle or Antioarticle Identity of the B Hadron at $t=0$}

In the determination of the level of statistics needed for detection of CP violation in the time distributions of the neutral B and B hadron decays, an essential step is the determination of the $t=0$ identity of the particle or antiparticle nature of the $B$ whose decay is to be examined for evidence of $C P$ violation. The $B$ and $B$ data samples must be separated so that a comparison between particle and antiparticle can be made. While a random assignment of B's into the particle or antiparticle 
categories will wash out any CP violation effect, a strategy to effect this separation need not be perfect to detect an asymmetry. To the level that any proposed strategy is not perfect in making separation into particle and antiparticle categories, the $C P$ violation effect will be lessened, more statistics will be required for the observation of it, and any observed effect will become harder to interpret.

There are several ways in which the identity of the B's at $t=0$ can be tagged. Among these are:

- It may be possible a $\mathrm{B}$ originates in the decay of a $\mathrm{B}^{*}$ resonance. The $B^{*}$ decay can serve to tag the identity of the $B$. In the particularly simple case of the decay $\mathrm{B}^{* \pm}>\mathrm{B}^{0} \pi^{ \pm}$, the sign of the $\pi^{ \pm}$will tag the $\mathrm{B}$ as $\mathrm{B}^{0}$ or $\mathbf{B}^{0}$.

- Observation of the sign of the lepton from the semileptonic decay of the other ("tag") B in the event. This technique is vulnerable to confusion and mistagging because of the $b->c->l e p t o n$ decays which produce a lepton of opposite charge from the lepton produced in the direct b->lepton decay. In addition, the mixing of the neutral B's will change particle to antiparticle and their subsequent semileptonic decays into the "wrong" charge lepton will also give erroneous tags.

- Direct observation of the charge of a $\mathrm{B}^{ \pm}{ }_{\mathrm{u}}$ associated with the "analysis" $B$ under study. This method is the least vulnerable to ambiguities but is difficult experimentally since it requires the complete reconstruction of the $\mathrm{B}^{ \pm}$. In the normal process of reconstructing the B it is easy to add or lose one charged track in the process of trying to reconstruct the secondary vertex.

In addition, there is a possibility that tagging may be avoided altogether. A region of phase 
space may be found where production of either the B or the $\mathrm{B}$ dominates so that the $\mathrm{B}$ (or $\mathrm{B}$ ) decay distribution can be observed. If differences are observed between this special region and the distributions as measured in other kinematic regions, then $C P$ violation may have been observed. The extraction of a CP violation phase from such data may be quite difficult since the relative production of $\mathrm{B}$ and $\mathrm{B}$ must be measured over a considerable kinematic range to allow such an extraction.

There may be other techniques that work for particular decays. However, what is almost certainly true is that all such tagging strategies require considerably higher statistics than an observation of time distributions for exclusive modes where the separation into B and $\mathrm{B}$ is not required.

\section{Yields of B Decavs in Exclusive Channels}

The final step in determining the sensitivity of a particular strategy for detecting (and measuring) CP violating effects is an estimate of the yields of exclusive decays which are expected to show sizable CP violation. Those yields must be decreased by the usual geometric and reconstruction efficiencies for both the "analysis" B and the "tag" B. Table III contains estimates of these yields for a few $J / \Psi$ decay modes which have significant levels of $C P$ violation. The geometric and detector efficiencies used in obtaining these yields are reasonable estimates based on the expectations and experiences of fixed target experiments (such as E771 ${ }^{17}$ ) and studies ${ }^{12,13.18}$ of the efficiencies achievable in SSC detectors.

Taking the numbers of produced $\mathrm{b} \mathrm{b}$ events given in Table I, the composite branching ratio, $7.9 \times 10^{-4}$ for the $\mathrm{J} / \Psi$ inclusive mode plus the subsequent $\mathrm{J} / \Psi->\mu \mu$ decay gives $1.2 \times 10^{5}$ inclusive $\mathrm{B}->\mathrm{J} / \Psi \rightarrow>\mu \mu$ events for a TEV II experiment and $1.6 \times 10^{8}$ events for an SSC experiment. Assuming $\mathrm{B}^{ \pm} \mathrm{u} / \mathrm{B}^{0} \mathrm{~d} / \mathrm{B}^{0}{ }_{\mathrm{s}}=2 / 2 / 1$ hadronization ratios, $4.8 \times 10^{4} / 4.8 \times 10^{4} / 2.4 \times 10^{4} \mathrm{~B}->\mathrm{J} / \Psi->\mu \mu$ of the various species are expected in a $10^{7} \mathrm{sec}$ TEV II experiment. For the SSC experiment we expect $6.4 \times 10^{7} / 6.4 \times 10^{7} / 3.2 \times 10^{7}$ such decays. We refer to Refs. 12 and 13 for a discussion of the problems associated with keeping even the relatively rare dimuon trigger rates low enough to allow all $\mathrm{B}->\mathrm{J} / \Psi->\mu \mu$ events to be written to tape. 
From the number of $\mathrm{B}->\mathrm{J} / \Psi->\mu \mu$ inclusive events produced in a $10^{7}$ second run, we can calculate the numbers of the exclusive decays in modes where CP violation is expected to be large. The number of produced events is given in the first and second column of Table III (using a $\varphi->\mathrm{K}^{+} \mathrm{K}^{-}$branching ratio of $50 \%$ and a $\mathrm{K}^{0} \mathrm{~s}^{->\pi^{+}} \pi^{-}$branching ratio of $68 \%$ ). The numbers represent the sum of both $B$ and $B$ decays. Under the assumption that the spectrometers at both TEV II and the SSC can achieve 30\% geometric acceptance, $90 \%$ trigger efficiency for dimuons, $60 \%$ efficiency for vertex cuts, and $80 \%$ efficiency for both detector and reconstruction efficiency, only approximately $13 \%$ of the produced events will be totally reconstructed. The number of reconstructed events expected in both kinds of configurations is given in the last two columns of Table III.

The added requirement of tagging with the other $B$ decay will further reduce the numbers of reconstructed events in the last two columns of Table III. Taking the most optimistic view of the single lepton tagging strategy (where the charge of the lepton from the semileptonic decay of the other B determines the particle or antiparticle nature of the "analysis" B) by ignoring the misidentification problems and assuming that perfect efficiency can be achieved for both electron and muon detection, we will still be able to tag only approximately $22 \%$ of the reconstructed events in Table III by this strategy alone. Indeed, even if all the tagging strategies mentioned above were to be employed, it is unlikely that a composite efficiency of $20 \%$ could be achieved. If it were possible to achieve $20 \%$, then the B or B data samples of a TEV II experiment for the exclusive modes listed in Table III might contain a few 10's of events each to use to search for CP violation. The SSC experiment would have a few thousand events in each time distribution.

It has been proposed ${ }^{31}$ that, since the sign of the $\mathrm{CP}$ violation can be determined for some exclusive decay modes, it may be possible to add data (with proper sign) from different decay modes to increase statistics. If this technique is feasible, several modes (such as the three given in Table III) could be added together increasing the TEV II statistics and, in that way, increase the sensitivity for observation of $\mathrm{CP}$ violating effects.

\section{Manifestations of CP Violation in B Decay}

The observation of $\mathrm{CP}$ violation in $\mathrm{B}$ decay is based on the observation of differences in the exclusive decays of $\mathrm{B}$ and $\mathrm{B}$ hadrons $\left(\mathrm{B}^{ \pm}{ }_{\mathrm{u}}, \mathrm{B}^{0}{ }_{\mathrm{d}}, \mathrm{B}^{0}{ }_{\mathrm{s}}, \mathrm{B}^{ \pm}{ }_{\mathrm{c}}\right.$ and the various baryonic states). These 
differences can be manifested either in differences in the integrated decay rate for a particular $B$ hadron relative to its antiparticle $B$ into a particular exclusive final state or in differences in the $B$ and $B$ time distributions. We will examine, in particular, the decay distributions of the neutral $B$ mesons. It is straightforward to write down the most general form of the proper time distribution for the decays of $\mathrm{B}^{0}$ and $\mathrm{B}^{0}$ into final states $f$ and $T$ where $f$ and $T$ are $C P$ conjugate. Using the nomenclature of ref 29 :

$$
\begin{aligned}
\Gamma\left(B^{0}{ }_{\text {phys }} \rightarrow 1\right)=\left|\left\langle i \mid B^{0}\right\rangle\right|{ }^{2} e^{-\gamma \tau} \mid \quad A^{2} & {\left[(\cos \Delta m \tau / 2)^{2}+|\lambda|{ }^{2}(\sin \Delta m \tau / 2)^{2}\right] } \\
& +A^{\cdot 2}\left[(\sin \Delta m \tau / 2)^{2}+\left.\lambda\right|^{2}(\cos \Delta m \tau / 2)^{2}\right] \\
+ & {\left[\lambda_{I} \sin \Delta m \tau-2 \lambda_{R^{A}} A^{\prime}\right] }
\end{aligned}
$$

and

$$
\begin{aligned}
\Gamma\left(\bar{B}_{\text {phys }} \rightarrow \bar{T}\right)=|\langle\hat{\mid} \mid \overline{B O}\rangle|{ }^{2} e^{-\gamma \tau_{1}} & A^{2} \\
& {\left[(\cos \Delta m \tau / 2)^{2}+|\bar{\lambda}|^{2}(\sin \Delta m z / 2)^{2}\right] } \\
& +A^{2}\left[(\sin \Delta m \tau / 2)^{2}+|\bar{\lambda}|^{2}(\cos \Delta m \tau / 2)^{2}\right] \\
+ & {\left.\left[\bar{\lambda}_{I} \sin \Delta m \tau-2 \bar{\lambda}_{R} A A^{\prime}\right]\right\} }
\end{aligned}
$$

In these expressions

$$
A=\left(e^{\Delta \gamma \tau / 4}+e^{-\Delta \gamma \tau / 4}\right) / 2 \cdot A^{\prime}=\left(e^{\Delta \gamma \tau / 4}-e^{-\Delta \gamma \tau / 4}\right) / 2
$$

and

$$
\lambda=(\mathrm{q} / \mathrm{p})^{\cdot}\langle\hat{\mathrm{f}} \mid \overrightarrow{\mathrm{B}} \mathrm{D}\rangle /\left\langle\mathrm{f} \mid \mathrm{B}^{0}\right\rangle \text { and } \lambda=(\mathrm{p} / \mathrm{q})^{\cdot}\langle\overline{\mathrm{T}} \mid \overrightarrow{\mathrm{B}}\rangle \mid\langle\hat{\mathrm{f}} \mid \overrightarrow{\mathrm{B}} \mathrm{0}\rangle
$$

Here, $\tau$ is the proper time, $\Delta \mathrm{m}$ is the difference in the masses and $\Delta \gamma$ is the difference in lifetimes of the light and heavy eigenstates of the $B$ mass matrix (in analogy with the $\mathrm{K}^{0}$ system). $C P$ violation is manifested in these formula by the difference between the parameters $\lambda$ and $\lambda$ or in a difference in the decay amplitudes $\left|\left\langle\rho \mid B^{0}\right\rangle\right|^{2}$ and $|\langle\hat{\imath} \mid \vec{\theta}\rangle|^{2}$. In analogy with the $K^{0}$ system, $p$ and $q$ are the coefficients of the superpositions of the light and heavy eigenstates of the $B$ mass matrix. $C P$ violation can either occur in the mass matrix ( $p$ is not equal to $q$ ) or in the decay amplitudes $\left(\left\langle f \mid B^{0}\right\rangle\right.$ is not equal to $\left.\left\langle\overline{7} \mid \bar{B}^{0}\right\rangle\right)$.

A considerable simplification occurs in these lifetime distributions if the lifetimes of the light and heavy eigenstates of the $B$ mass matrix are the same. This is expected for the B system in marked contrast to the $\mathrm{K}$ system where the lifetimes of the $\mathrm{K}^{0}{ }_{1}$ and $\mathrm{K}^{0}{ }_{2}$ are very different. Setting 
$\Delta \gamma=0$, we obtain $A=1, A^{\prime}=0$ and

$$
\begin{aligned}
& \Gamma\left(B_{\text {phys }}^{0} \rightarrow f\right)=\left|\left\langle f \mid B^{0}\right\rangle\right|{ }^{2} \theta^{-\gamma} \tau_{\left\{(\cos \Delta m \tau / 2)^{2}+|\lambda|{ }^{2}(\sin \Delta m \tau / 2)^{2}+\lambda_{I} \sin \Delta m \tau\right\}} \\
& \left.\Gamma\left(\bar{B}_{\text {phys }} \rightarrow \bar{T}\right)=\left.\left|\left\langle\bar{f} \mid \overline{B^{0}}\right\rangle\right|{ }^{2} \theta^{-\gamma \tau}\left|(\cos \Delta m z / 2)^{2}+\right| \bar{\lambda}\right|^{2}(\sin \Delta m z / 2)^{2}+\lambda l \sin \Delta m \tau\right\}
\end{aligned}
$$

These time distributions can be integrated (either for all $\tau$ or to a specific $\tau$ chosen to maximize the integrated asymmetry between the $\mathrm{B}$ and $\mathrm{B}$.

The values of $\lambda$ and $\lambda$ (as well as the other decay amplitudes) are functions of the elements of the Kobayashi -Maskawa matrix and, therefore, can be estimated (within the Standard Model framework) from the present experimental measured values of that decay matrix. Table IV below gives the expected asymmetries for several experimentally accessible $\mathrm{J} / \Psi$ decays which are expected to show large CP asymmetries.

For purposes of simplification to show the differences that $\mathrm{CP}$ violation can generate in the $B$ and $B$ decay distributions, let us specialize these lifetime distributions one step further to the case of exclusive decays in which the final state $f$ is an eigenstate of CP. In that case, it can be shown that $|\lambda|^{2}=|\bar{\lambda}|^{2}=1$ and $\lambda_{I}=-\bar{\lambda}_{I}$. This type of decay mode $\left(B \rightarrow \psi \psi K_{S}^{0}, \psi \Phi\right.$, etc.) then exhibits a particularly simple and elegant signature of $\mathrm{CP}$ violation

$$
\begin{aligned}
& \Gamma\left(B_{\text {phys }}^{0}{ }^{-} f\right)=\left|\left\langle f \mid B^{0}\right\rangle\right|{ }^{2} e^{-\gamma z}\left\{1+\lambda_{I} \sin \Delta m z\right\} \\
& \left.\Gamma\left(\bar{B}_{\text {phys }} \rightarrow \bar{T}\right)=\mid\left\langle\left.\overrightarrow{T|\bar{B}\rangle}\right|^{2} \theta^{-\gamma \tau}\right| 1-\lambda_{I} \sin \Delta m \tau\right\}
\end{aligned}
$$

The mixing oscillations of the $B$ and the $B$ decays are shifted by $180^{\circ}$ with respect to each other. $\mathrm{CP}$ violation can be observed either by integrating these distributions out to an appropriate point chosen to maximize the differences in integrated rates or by a bin by bin comparison of the $B$ and $B$ time distributions.

In Fig. $3 \mathrm{a} \quad \mathrm{B}^{0}{ }_{\mathrm{d}}$ and $\mathrm{B}^{0} \mathrm{~d}$, mixing $(\Delta \mathrm{m} / \gamma=0.78)$ oscillations are shown (with the exponential part of the decay distribution multiplied out) for a CP eignestate exclusive decay mode in which the CP violation is "maximal", that is where $\lambda_{I}=1$, i.e., $\lambda$ is totally imaginary. We have 
assumed that a tagging strategy can be concocted which perfectly separates the $B$ decays into $B(t=0)$ and $B(t=0)$ categories. The error bars shown for the $B$ decays are those appropriate for a 1000 event sample and include the depletion of events near $t=0$ caused by the vertex experimental cut and the gradual loss of statisitics at large proper time due to the exponential part of the decay distribution. The vertex cut, which requires that the secondary vertex be separated by 50 microns from the primary vertex in the plane transverse to the beam, is made to insure that the secondary vertex is real and not caused by mismeasurements of tracks from the primary vertex. The effect of this cut is shown in Fig $3 d$ as an efficiency verses proper time.

As can be seen in Fig. 3a, 1000 events are completely adequate to see differences between the $\mathrm{B}^{0}{ }_{\mathrm{d}}$ and $\mathrm{B}_{\mathrm{d}}^{0}$ time distributions when $\lambda_{\mathrm{I}}=1$. In Fig. $3 \mathrm{~b}$ the time distribution is shown for the case where $\lambda_{I}=.3$ and $\lambda_{R}=0.95$ (more nearly the values expected for the $B->\Psi K^{0}{ }_{s}$ decay mode). The $B$ and $B$ distributions are still quite distinct and $C P$ violation can be detected quite easily. As the imaginary part approaches zero, the time distributions become more and more nearly exponential, the distinction between $\mathrm{B}$ and $\mathrm{B}$ distributions gradually disappears and higher and higher levels of statistics are necessary to detect $C P$ asymmetries.

Finally, Fig. $3 \mathrm{c}$ shows the time distribution for $\mathrm{B}^{0}{ }_{\mathrm{s}}$ decays (with maximal $\mathrm{CP}$ violation). The mixing in the $\mathrm{B}^{0}{ }_{\mathrm{S}}$ system is expected on fairly general grounds to be six times the size of the $\mathrm{B}^{0} \mathrm{~d}$ mixing. Provided that a resolution in the measurement of proper time of approximately $10^{-13}$ seconds can be obtained, the $\mathrm{B}^{0}{ }_{s}$ time distribution should be spectacular, going through several oscillations within the range of proper time where data can be obtained.

\section{Conclusions}

A few hundred reconstructed events in a few selected exclusive modes will be required to detect and study CP violation in B- $>\mathrm{J} / \Psi$ decays. Production of $>100$ million $\mathrm{b} \sigma$ events (implying operation at $10^{7}$ interactions per second) will be required to achieve a few hundred tagged reconstructed events in the particular $J / \Psi$ modes where $C P$ violation effects are supposed to be large. This appears to be be on the edge of what might (optimistically) be expected for experiments based on the $J / \Psi$ trigger/tagging strategy at the present accelerators. To improve the chances for observation of $C P$ violation using such a strategy, experiments must aim at operating points 
considerably higher than $10^{7}$ interactions per second. However, even at luminosities below $10^{32}$ $\mathrm{cm}^{-2} \mathrm{sec}^{-1}$, this strategy appears to produce $B$ decay data samples large enough to put $C P$ observation within the reach of experiments at the SSC. Other strategies such as those based on triggering on the "tag" semileptonic B decays should be investigated to see if larger data samples pertinent to the observation of $\mathrm{CP}$ violation can be accumulated in this manner. 


\section{References}

1.E. Bloom, "Very High Luminosity $\mathrm{e}^{+} \mathrm{e}^{-}$Colliders for b, c, Quark and Tau Lepton Phyiscs, The PEP Option", B-Meson Factory Workshop, SLAC (Sept, 1987).

2. Review of Particle Properties, Phys. Lett. 170B 15(1986) .

3. H.Albrecht et al.,Phys. Lett. 192B,245(1987).

4. J.P. Albanese et al. (WA75 Collaboration), Phys. Lett. 158B, 186(1985).

5. K. Berkelman, invited talk, to be published in the Proceedings of the B Physics Workshop, SLAC, (Sept.,1987).

6. M.G. Catanesi et al. (WA78 Collaboration), Phys. Lett. 187B, 431(1987).

7. M.G. Catanesi et al. (WA78 Collaboration), CERN-EP/87, (Nov, 1987).

8. C. Albajar et al. (UA1 Collaboration), Phys. Lett. 186B, 237(1987).

9. P. Boralo et al. (NA10 Collaboration), presented in the EPS Int. Conf. on High-Energy Physics, Uppsala, 1987.

10. E. Berger, "Heavy Quark Production in Hadron Collisions", ANL-HEP-PR-87-53, (June,1987); E. Berger, "Momentum Correlations in Heavy Quark Hadroproduction", ANL-HEP-PR-87-113, (Nov.,1987); E. Berger, talk given at Fermilab Beauty Workshop, (Nov.,1987).

11. E.Berger, J.C.Collins, D.E.Soper, Proceedings of the 1986 Summer Study on the Physics of the Superconducting Collider, Snowmass,54(1986). 
12. B. Cox, F. Gilman and T. Gottshalk, Proceedings of the 1986 Summer Study on the Physics of the Superconducting Collider, Snowmass,33(1986).

13. B.Cox and D.E. Wagoner, Proceedings of the 1986 Summer Study on the Physics of the Superconducting Collider, Snowmass,83(1986).

14. B.Cox, "Beauty Physics at the Ultrahigh Energies of the ELOISATRON", to be published in the Proceedings of the ELOISATRON Workshop on Physics at Ultrahigh Energies, Erice, (June,1987).

15. H.U.Bengtsson and G. Ingelman, Computer Phys. Comm. 34,(1985)351.

16. K.Foley et. al., "Report of the Intermediate-p ${ }_{\perp}$ Detector Group: A Beauty Spectrometer for the SSC", to be published in the Proceedings of the Berkeley Workshop on Detectors for the SSC, Berkeley, (July, 1987).

17. B.Cox et al., "A Proposal to Study Beauty Production and Other Heavy Quark Physics Associated With Dimuon Production in 800-925GeV/c PP Interactions, Fermilab Proposal 771, (April, 1986).

18. S.Conetti,B.Cox,W.Selove and D.E.Wagoner, "A Single Muon Trigger for E771", to be published in the Proceedings of the Fermilab Beauty Workshop, (Nov., 1987).

19. R.J. Morrison, S. McHugh and M.V. Purohit, "A Semileptonic Electron Trigger for Recording $\mathrm{B}^{0}, \mathrm{~B}^{0}$ Events", UCSB-HEP-87-14,to be published in the Proceedings of the Berkeley Workshop on Detectors for the SSC, (July, 1987).

20. L.Rossi et al., "Proposal for a Test of an Impact Parameter Trigger Aimed at a High Statistics Heavy Quark Study", CERN/SPSC 85-62,SPSC/P218,(Oct,1985).

21. W. Selove, "A Multiplicity Change Trigger for Fermilab Experiment E771", to be published in the Proceedings of the Fermilab Beauty Workshop, (Nov.,1987). 
22. C. Fisher et al.,"A Proposal to Study the Production and Decay Properties of Beauty Flavoured Hadrons", CERN/SPSC 87-2, SPSC/P 226, (Jan.,1987).

23. K.Berkelman, invited talk, to be published in the Proceedings of the Stanford Symposium on Heavy Flavors, Stanford University, (September,1987).

24. P.Haas et al., CLEO Collaboration, Phys. Rev. Lett. 55, 1248(1985).

25. Proceedings of the Workshop on Triggering, Data Acquisition and Computing for High Energy/High Luminosity Hadron-Hadron Colliders, edited by B. Cox, R. Fenner, P.Hale, Fermilab, (Nov.,1985).

26. Private communications, ARGUS and CLEO Collaborations; also certain of the quoted branching ratios were presented in the EPS Int. Conf. on High-Energy Physics, Uppsala, 1987.

27. J.D. Bjorken, Estimates of Decay Branching Ratios for Hadrons Containing Charm and Bottom Quarks, unpublished, (July,86).

28. J.D. Bjorken, "Rare B-Decays:Experimental Prospects and Problems",Fermilab-Conf-87/83, 2025.000, (May,1987).

29. J. Rosner and I. Dunietz, Physical Review 34, 1404 (1987).

30. I. Bigi and A.I. Sanda, "CP Violation in Heavy Flavor Decays: Predictions and Search Strategies", SLAC-Pub-3949, (May,1986).

31. I. Bigi, "Mixing and CP Violation in B and D Decays-Future Search at Hadron Machines", SLAC-Pub-4000, (June,1986).

32. K.L. Foley et al., "Bottom and Top Physics", SLAC-Pub-4426, to be Published in the Proceedings of the Berkeley Workshop on Detectors for the SSC, (July, 1987). 


\section{Table I* \\ Compariscon of Beauty \\ Hadmonduction at Difierent \\ Machines}

TEY II

TEY J

SSC

$\sqrt{\mathrm{s}}(\mathrm{TeV})$

.041

1.8

40

$\sigma(\mathrm{bb})\left(\mathrm{cm}^{2}\right)$

$\approx 2.4 \times 10^{-32}$

$=1.5 \times 10^{-20}$

$\approx 1.0 \times 10^{-28}$

$\sigma(\mathrm{b} \sigma) / \sigma_{\mathrm{T}}(\mathrm{pN})$

$\approx 0.75 \times 10^{-8}$

$=1.5 \times 10^{-4}$

$\approx 10^{-3}$

$\approx 0.75 \times 10^{8}$

$\approx 1.5 \times 10^{9}$

$\approx 10^{11}$

$\approx 10^{14}$

$\approx 10^{13}$

$\approx 10^{14}$

$\left\langle\mathrm{p}_{\mathrm{b}}\right\rangle<45^{\circ}$

$145 \mathrm{GeV} / \mathrm{c}$

$38 \mathrm{GeV} / \mathrm{c}$

$32 \mathrm{GeV} / \mathrm{c}$

$\approx 100$

$\approx .2 \mathrm{~cm}$
$130 \mathrm{GeV} / \mathrm{c}$ $60 \mathrm{GeV} / \mathrm{c}$

$\approx 35$

$\approx .3 \mathrm{~cm}$

* For purposes of estimating the detector dependent entries in this table,the detectors for TEV II (Fermilab Experiment E771 17 is taken as a model) and the SSC ${ }^{12.10}$ have been taken to be relatively forward along a given beam direction. Because of the low momentum and wide angular distribution of the B hadrons at TEV I, the TEV I detector has been assumed to be a $4 \pi$ detector. The calculation of the average momentum of the $b$ quark has been done for b's in an angular cone of $45^{\circ}$ around the beam direction for all three experimental configurations. 


\section{Table II}

$B \rightarrow \mathrm{I} / \Psi$. $\Psi^{\prime}$, Semileptonic Decay Modes*

$$
\begin{array}{ll}
\mathrm{B} \longrightarrow \mathrm{J} / \Psi+\mathrm{x} & (1.2 \pm 0.3 \%) \\
\mathrm{B} \longrightarrow \Psi^{\prime}+\mathrm{x} & (0.46 \pm 0.3 \%) \quad \text { experimental branching ratios } \\
\mathrm{B} \longrightarrow \mu \nu+\text { hadrons } & (11.0 \pm 0.9 \%)
\end{array}
$$

\begin{tabular}{|c|c|}
\hline $\mathrm{B}_{\mathrm{u}}^{ \pm} \longrightarrow \Psi \mathrm{K}^{ \pm}$ & $0.10 \%$ \\
\hline $\mathrm{B}^{ \pm}{ }_{\mathrm{u}}^{-} \rightarrow \Psi \mathrm{K}^{ \pm} \pi^{+} \pi^{-}$ & $0.10 \%$ \\
\hline $\mathrm{B}_{\mathrm{u}}^{ \pm} \rightarrow \Psi \mathrm{K}^{0} \pi^{ \pm}$ & $0.35 \%$ \\
\hline $\mathrm{B}^{ \pm}{ }_{\mathrm{u}} \longrightarrow \Psi^{\prime} \mathrm{K}^{ \pm}$ & -..-- \\
\hline$\rightarrow \boldsymbol{\psi} \mathrm{K}^{+} \pi^{-}$ & $0.25 \%$ \\
\hline$\rightarrow \Psi \mathrm{K}^{0}$ & $0.07 \%$ \\
\hline $\mathrm{B}^{0} \mathrm{~d}^{0} \longrightarrow \psi \mathrm{K}^{0} \pi^{+} \pi^{-}$ & $0.07 \%$ \\
\hline$\rightarrow \Psi \pi^{+} \pi^{-}$ & $0.01 \%$ \\
\hline$\rightarrow \Psi \mathrm{K} * 0$ & $\cdots$ \\
\hline$\rightarrow \Psi \mathrm{K}^{+} \mathrm{K}^{-}$ & $0.05 \%$ \\
\hline $\mathrm{B}^{0}{ }_{\mathrm{S}} \rightarrow \Psi \mathrm{K}^{+} \mathrm{K}^{-} \pi^{+} \pi^{-}$ & $0.05 \%$ \\
\hline $\mathrm{B}_{\mathrm{s}}{ }_{\mathrm{s}} \longrightarrow \Psi \mathrm{K}^{0} \overline{\mathrm{K}}^{0}$ & $0.05 \%$ \\
\hline $\mathrm{B}^{0}{ }_{\mathrm{s}} \longrightarrow \Psi \mathrm{K}^{0} \overline{\mathrm{K}}^{0} \pi^{+} \pi^{-}$ & $0.05 \%$ \\
\hline $\mathrm{B}^{0}{ }_{\mathrm{S}} \longrightarrow \Psi \mathrm{K}^{+} \overline{\mathrm{K}^{0}} \pi^{-}$ & $0.15 \%$ \\
\hline $\mathrm{B}_{\mathrm{S}}^{0} \rightarrow \Psi \mathrm{K}^{-} \mathrm{K}^{0} \pi^{+}$ & $0.15 \%$ \\
\hline $\mathrm{B}_{\mathrm{s}}^{0} \rightarrow \Psi \varphi$ & $0.30 \%$ \\
\hline$B_{c}^{ \pm} \rightarrow \Psi \pi^{ \pm}$ & $0.60 \%$ \\
\hline$B_{c}^{ \pm} \longrightarrow \psi \pi^{ \pm} \pi^{-} \pi^{+}$ & $0.40 \%$ \\
\hline$\pm{ }_{c} \longrightarrow \Psi \pi^{ \pm} \pi^{-} \pi^{+} \pi^{-} \pi^{+}$ & $0.20 \%$ \\
\hline
\end{tabular}

$\Psi \cdot \Psi^{\prime}$ Exclusive Decays
Exp.Measurements ${ }^{23.28}$ Experiment

(where available)

$0.09 \% / 0.07 \%$

$0.11 \%$

$-\cdot-\cdots-\cdot$

$0.22 \%$

$<0.63 \%$
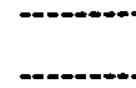

$0.41 \% / 0.33 \%$

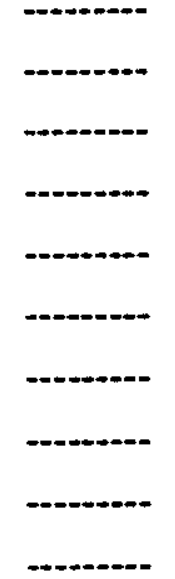

ARGUS
CLEO

ARGUS

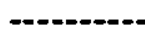

CLEO/ARGUS

CLEO/ARGUS

ARGUS
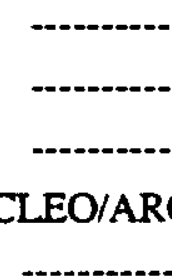
Table III

Yields of B Exclusive Decavs $/ 10^{7}$ sec

\begin{tabular}{|c|c|c|c|c|}
\hline Exclusive Decays & $\begin{array}{l}\text { \#Produced } \\
\text { TEV II }\end{array}$ & $\begin{array}{l}\text { \#Produced } \\
\text { SSC }\end{array}$ & $\begin{array}{l}\text { \#Reconst. } \\
\text { TEV II }\end{array}$ & $\begin{array}{l}\text { \#Reconst. } \\
\text { SSC }\end{array}$ \\
\hline $\begin{array}{r}\mathrm{B}^{0} \mathrm{~s}^{->\psi+\varphi} \\
\longrightarrow \mathrm{K}^{+} \mathrm{K}^{-}\end{array}$ & 3200 & $4.2 \times 10^{8}$ & 420 & $5.5 \times 10^{5}$ \\
\hline $\begin{array}{r}\mathrm{B}^{0} \mathrm{~d}^{-}>\psi+\mathrm{K}_{\mathrm{S}}^{0} \\
\longrightarrow \rightarrow \pi^{+} \pi^{-}\end{array}$ & 2000 & $2.7 \times 10^{8}$ & 260 & $3.5 \times 10^{5}$ \\
\hline $\mathrm{B}^{0} \mathrm{~d}^{->}+\Psi+\pi^{+} \pi^{-}$ & 400 & $5.7 \times 10^{5}$ & 50 & $7.4 \times 10^{4}$ \\
\hline
\end{tabular}




\section{Tabie IY}

\section{Exoected CP Violation Asymmetries}

\section{In Various Exclusive B Decay Modes}

$L^{*}$. clusive Decay Mode

Expected Asymmetry*

Ref.29 Ref.30.31 Ref.32 Ref 28

$\mathrm{B}^{0}{ }_{\mathrm{d}} \longrightarrow \psi \mathrm{K}^{0}$

$8 \%$

$2-20 \%$

$5-30 \%$

$B^{0}{ }_{d} \longrightarrow \Psi K^{0} \pi^{0}$

$-. \quad 2-20 \%$

5-30\%

$\mathrm{B}^{0} \mathrm{~d} \longrightarrow \Psi \mathrm{K}^{0} \pi^{+} \pi^{-}$

$--$

$B^{0}{ }^{0} \longrightarrow \psi \varphi$

$2-20 \%$

$5-30 \%$

$B^{0} \mathrm{~d} \longrightarrow \Psi \pi^{0}$

$8 \%$

-...

$\mathrm{B}^{0} \mathrm{~d}^{\mathrm{d}} \longrightarrow \Psi \pi^{+} \pi^{-}$

-.- $2-20 \%$

$\mathrm{B}_{\mathrm{s}}{ }_{\mathrm{S}} \rightarrow \Psi \varphi$

$-2 \%$

$0.1-1.0 \%$
$-\quad 0.1-1.0 \%$

$\mathrm{B}_{\mathrm{S}} \mathrm{S} \longrightarrow \Psi \eta \pi$

-.. $\quad 0.1-1.0 \%$

*Some of these estimates (Refs. 29,30,31) were published prior to the announcement of the large $\mathrm{B}^{0} \mathrm{~d}$ mixing by the ARGUS collaboration. 


\section{Eigures}

Fig. 1a Correlation of momentum of the b quark with production angle for TEV II, TEV I, and the SSC

Fig. Ib Average momentum for electrons from the semileptonic decay of B hadrons at TEV II, TEV I and the SSC.

Fig. 2a Correlation of production angle of the $b$ quark with production angle of the $\bar{\sigma}$ quark at SSC.

Fig. $2 b$ Correlation of production angle of the $b$ quark with production angle of the $\bar{\sigma}$ quark at TEV I.

Fig. $2 c$ Correlation of production angle of the $b$ quark with production angle of the $\bar{b}$ quark at TEV II.

Fig. 3a Time distribution for $\mathrm{B}^{0}{ }_{j}$ and $\mathrm{B}^{0}{ }_{j}$ decays $\left(\lambda_{i}=1.0, \Delta \mathrm{m} / \gamma=0.78\right)$

Fig. 3b Time distribution for $\mathrm{B}^{0}{ }_{d}$ and $\mathrm{B}^{0}{ }_{d}$ decays $\left(\lambda_{i}=0.3, \Delta \mathrm{m} / \gamma=0.78\right)$

Fig. 3c Time distribution for $\mathrm{B}^{0}{ }_{S}$ and $\mathrm{B}^{0}{ }_{s}$ decays $\left(\lambda_{i}=1.0, \Delta \mathrm{m} / \gamma=5.0\right)$

Fig. 3d Efficiency vs. proper time due to vertex cut on B decay secondary vertices. 
pig. 1a

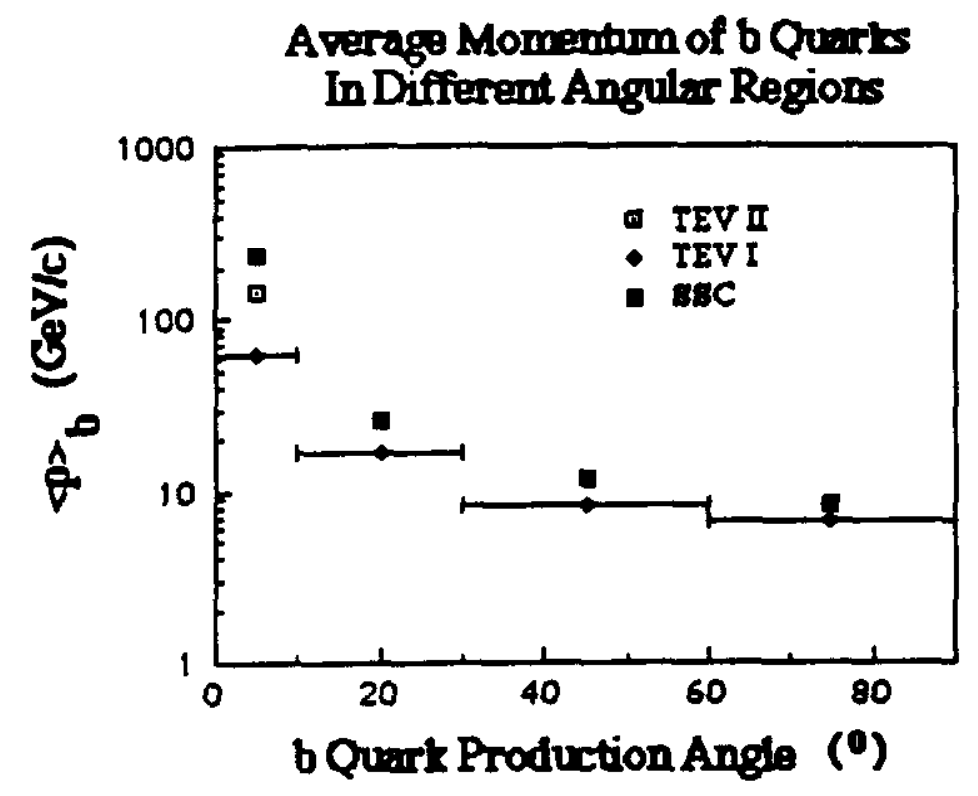

Fis. 16

Arerage Electron Momentam

$\mathrm{B} \rightarrow \mathrm{Dev}$

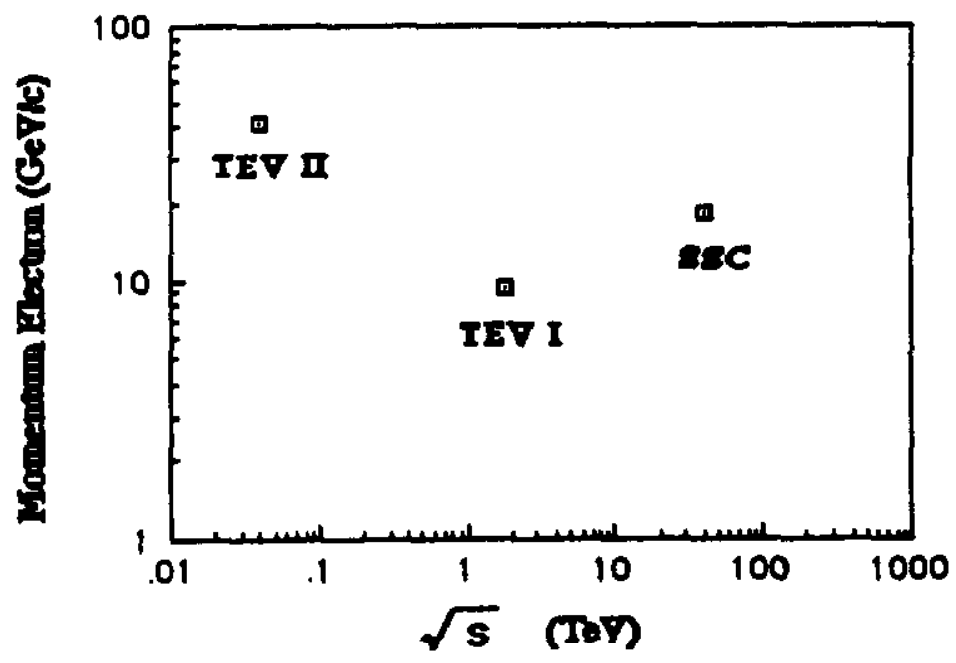


Fie. 2a

SSC

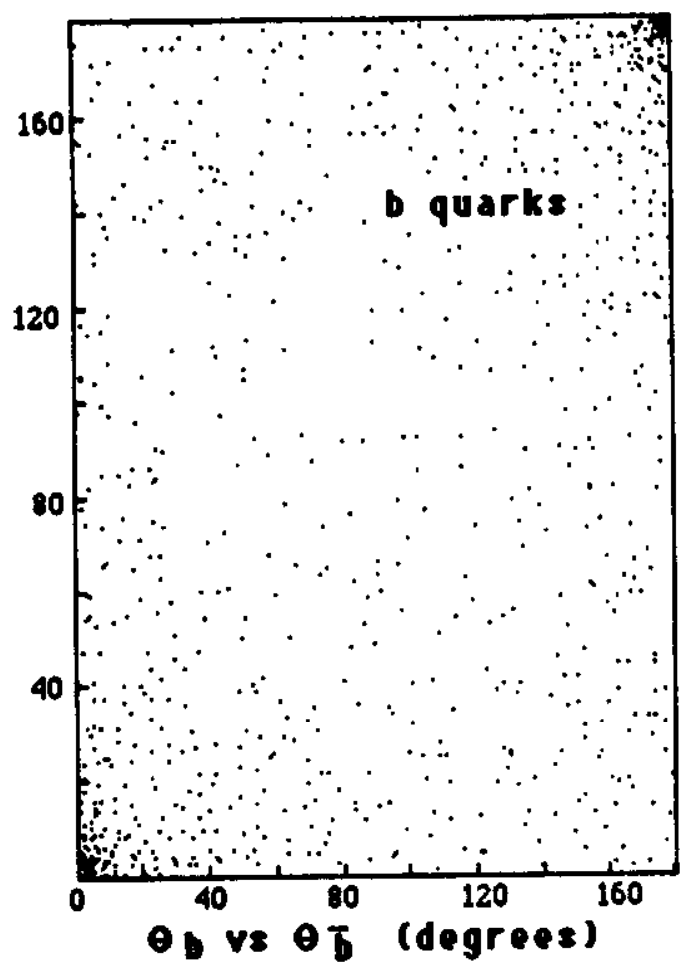

Fis. 20

TEV I

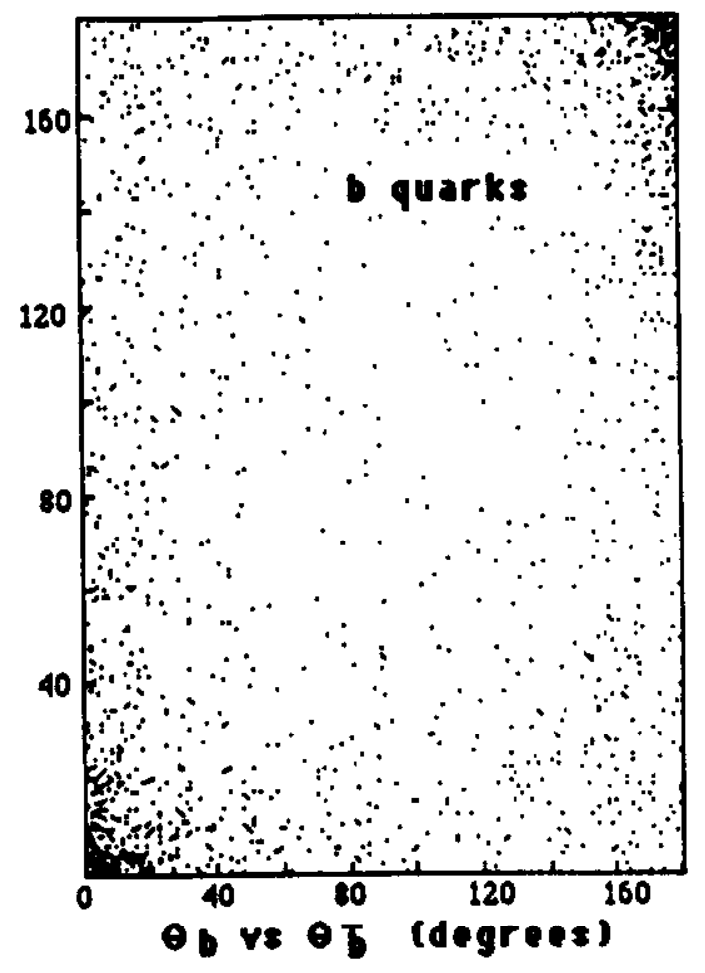

Fie. 2c

TEY II

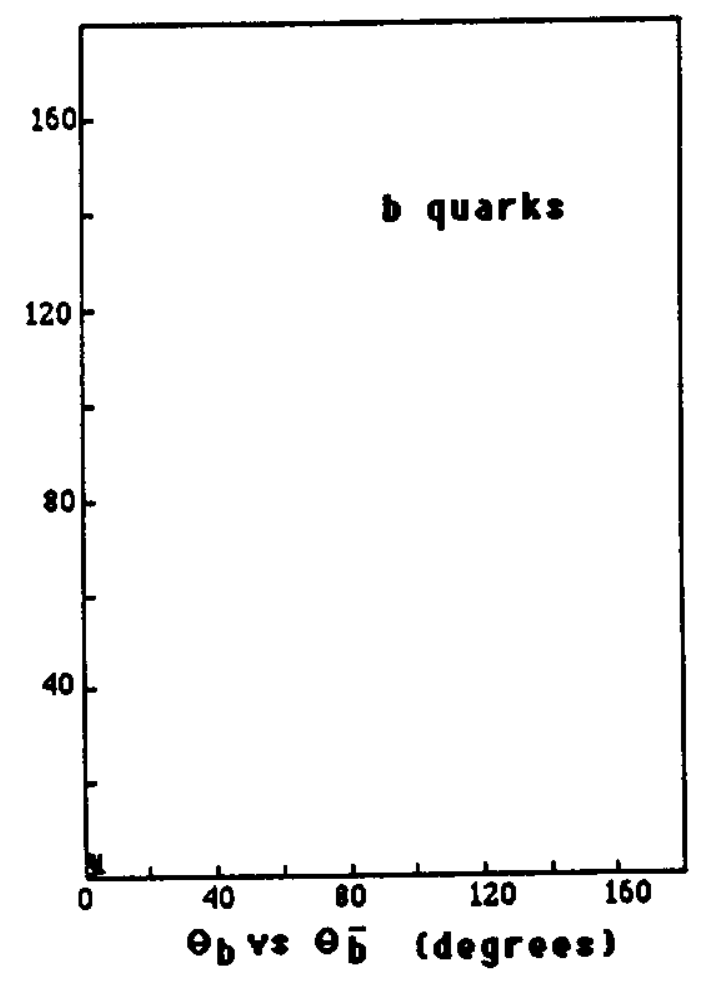


74. 30

B $_{\text {d }}^{\circ}$ Dees Tim Distibution

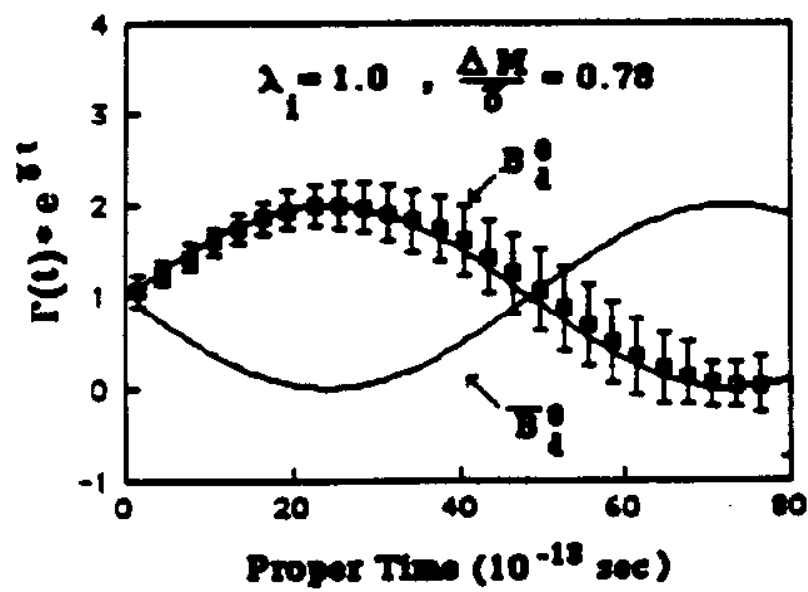

F5. 3e

$B_{s}^{0}$ Decay Time Distibution

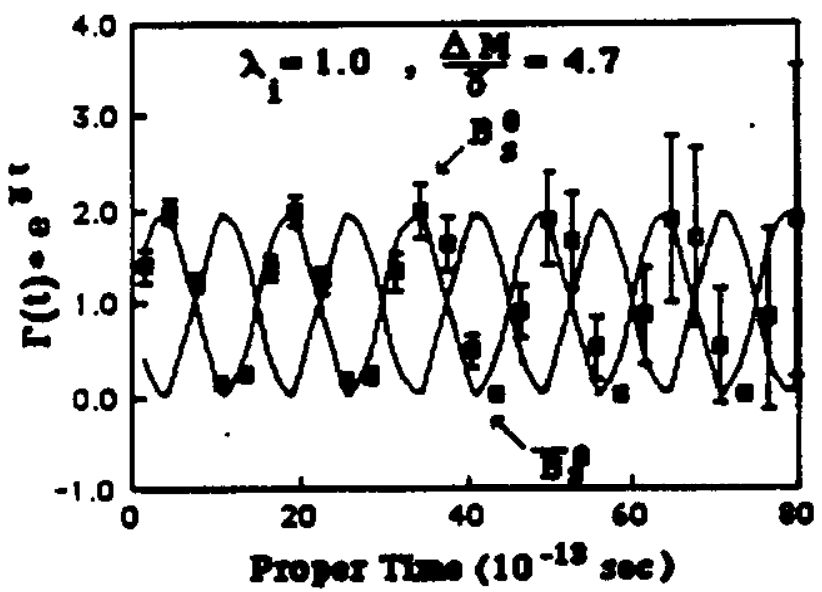

F2. 30

B9 Dees Tim Distibution

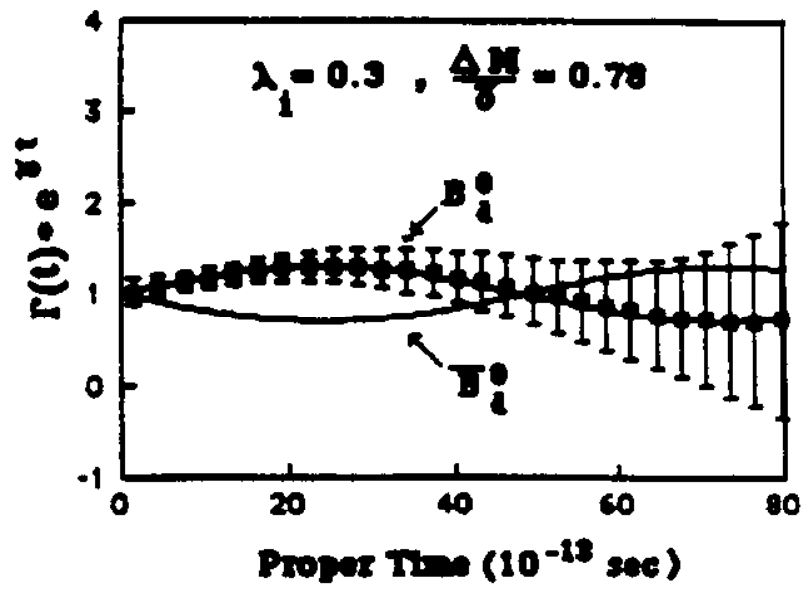

F2. 34

Secondary Yortax Cut Emicioney

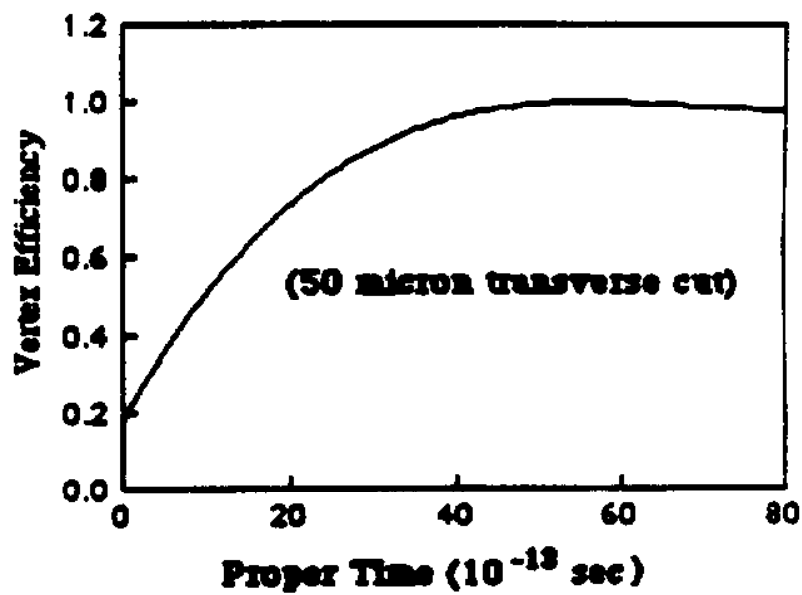

